\title{
Spin-vibronic superexchange and dynamical vibronic order in ammoniated cubic fullerides
}

\author{
L.F. Chibotaru \\ Division of Quantum and Physical Chemistry, University of Leuven, Celestijnenlaan 200F, B-3001 Leuven, Belgium \\ Received 7 December 2006; accepted 25 December 2006 \\ Available online 21 January 2007
}

\begin{abstract}
In the Mott-Hubbard cubic fulleride $\mathrm{Li}_{3}\left(\mathrm{NH}_{3}\right)_{6} \mathrm{C}_{60}$ the superexchange energy is found to be much smaller than the rotational quantum for Jahn-Teller deformations at fullerene sites. This gives rise to a new type of superexchange interaction involving threefold degenerate vibronic ground states of $\mathrm{C}_{60}^{3-}$ ions. Comparison of the spin-vibronic spectrum for a dimer fragment $\left[\mathrm{C}_{60}^{3-}\right]_{2}$ of a b.c.c. lattice with the spin-orbital spectrum of a corresponding dimer containing threefold orbitally degenerate sites $\left(t_{2 g}^{1}-t_{2 g}^{1}\right.$ model) exhibits drastic differences. In contrast to spin-orbital models, the spin-vibronic superexchange can only be antiferromagnetic and shows significant vibronic reduction of superexchange amplitude in agreement with magnetic susceptibility data. In function of transfer parameters two quadrupolar fully dynamical vibronic orders with quenched vibronic moments on sites develop in the ground state. The spectrum of collective vibronic excitations is calculated and the stability of vibronic ordered phases is discussed.
\end{abstract}

(c) 2007 Elsevier B.V. All rights reserved.

Keywords: Superexchange interaction; Dynamical Jahn-Teller effect; Mott-Hubbard fullerides

\section{Introduction}

Alkali doped fullerides have been the object of intensive investigations in the last decade [1,2]. In these crystals the electrons from alkali atoms are transferred into threefold degenerate orbitals of the fullerene sites. In the Mott-Hubbard insulating regime, the relatively strong intrasite electron repulsion together with orbital degeneracy results in a concomitant presence of spin, orbital and lattice (JahnTeller) degrees of freedom in the low-energy states. In this respect alkali doped fullerides are similar to other strongly correlated materials with orbital degeneracy [3,4]. A remarkable feature of fullerides is that the band energy, the intrasite Jahn-Teller effect and the frequency of Jahn-Teller modes are within the same energy scale. As a result the Jahn-Teller effect in fullerides is manifested quite differently from other materials.

E-mail address: Liviu.Chibotaru@chem.kuleuven.be
Recently, the first cubic Mott-Hubbard fulleride, $\mathrm{Li}_{3}\left(\mathrm{NH}_{3}\right)_{6} \mathrm{C}_{60}$, has been obtained and investigated [5]. In contrast to other ammoniated fullerides [6,7], in this compound each fullerene site is in a crystal field of cubic symmetry preserving the threefold degeneracy of the LUMO orbitals. The magnetic susceptibility was found to be in a full agreement with an isotropic $S=1 / 2$ Heisenberg model for a b.c.c. lattice [5]. This is neither consistent with a situation of undistorted fullerenes, where a Hund state $(S=3 /$ 2 ) is realized at each site, nor it is the case of static JahnTeller distortions as evidenced by structural data [5]. There is therefore a strong indication that dynamical Jahn-Teller effect plays an important role in the low energy phase of this compound.

Here we address the origin of the ground state and lowlying excitations in $\mathrm{Li}_{3}\left(\mathrm{NH}_{3}\right)_{6} \mathrm{C}_{60}$. It follows that the intermediate vibronic coupling and the local cubic symmetry at fullerene sites ensures that vibronic dynamics of individual $\mathrm{C}_{60}^{3-}$ anions is not quenched. The localized spins reside in threefold degenerate vibronic ground states of fullerenes thus giving rise to spin-vibronic superexchange between 
fullerene sites [8]. Such manifestation of Jahn-Teller effect, which is essentially dynamic in the present case, is due to the molecular nature of the crystal and does not occur in insulating perovskites with orbitally degenerate sites, where the Jahn-Teller effect is either quenched or static $[9,10]$.

\section{Jahn-Teller dynamics on individual $\mathrm{C}_{60}^{3-}$ sites in a cubic lattice}

The partly occupied orbitals $t_{1 u} \gamma, \gamma=x, y, z$ at each fullerene site interact with fivefold degenerate $H_{g}$ vibrations of the corresponding fullerene giving rise to vibronic $t \otimes H$ problem [11,12]. In the case of one or two electrons, the $t \otimes H$ interaction splits the $t_{1 u}$ shell into the ground nondegenerate and the excited twofold degenerate levels. The minima on the ground energy surface form a twofold continuum (two-dimensional trough) $[11,12]$. The third added electron occupies the degenerate orbitals, which split as well in virtue of the Jahn-Teller theorem. The resulting adiabatic potential has a three-dimensional trough of minima in the ground state [13]. Actually there are eight modes of $H_{g}$ vibrations in $\mathrm{C}_{60}, \omega_{\mu H}=271-1575 \mathrm{~cm}^{-1}$. The low-lying vibronic states can be conveniently described by an effective one-mode problem with the frequency $\bar{\omega}_{H}=\sum_{\mu} p_{\mu} / \sum_{\mu}\left(p_{\mu} / \omega_{\mu H}\right)$, where $p_{\mu}$ is the percent contribution of the $\mu$ th mode to the energy of Jahn-Teller stabilization $\left(E_{\mathrm{JT}}\right)$ [14]. Using for $p_{\mu}$ the data extracted from photoemission of $\mathrm{C}_{60}^{-}[15]$ we obtain $\hbar \bar{\omega}_{H}=600 \mathrm{~cm}^{-1}[16]$. With current estimation of the Jahn-Teller energy for $\mathrm{C}_{60}^{3-}, E_{\mathrm{JT}}=0.15 \mathrm{eV}$ [18], we have the ratio $E_{\mathrm{JT}} / \hbar \bar{\omega}_{H}=2$. corresponding to intermediate vibronic coupling [12].

The electron interaction in the $t_{1 u}$ shell is described by the intra $\left(U_{\|}\right)$and interorbital $\left(U_{\perp}\right)$ repulsion and the Hund's rule coupling $(J)$, related by $U_{\|}-U_{\perp}=2 J$. For pristine fullerene crystals $U_{\|} \approx 1-1.5 \mathrm{eV}$ and $J \approx 0.03$ $0.05 \mathrm{eV}$ [1]. In doped crystals $U_{\|}$is further reduced by the effects of screening. In $\mathrm{C}_{60}^{3-}$ there is a competition between Hund's rule coupling, which tends to align the three electrons ferromagnetically (Fig. 1a), and the Jahn-Teller effect which tends to populate consecutively the nondegenerate orbital levels (Fig. 1b). To compare the energies of these two electronic configurations we should take also into account the change of vibrational energy in the Jahn-Teller configuration [12]. Thus we have $3 U_{\|}-9 J+5 / 2 \hbar \bar{\omega}_{H}$ for the Hund's configuration and $3 U_{\|}-4 J-E_{\mathrm{JT}}+\hbar \bar{\omega}_{H}$ for the Jahn-Teller configuration. The gain of $3 / 2 \hbar \bar{\omega}_{H}$ in the

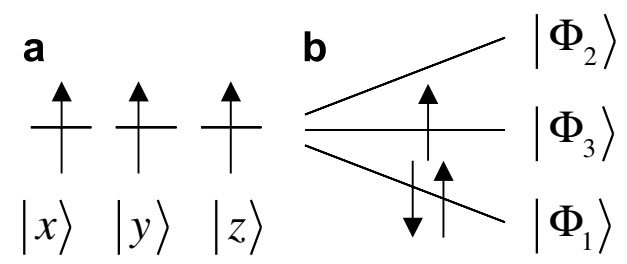

Fig. 1. Hund's (a) and Jahn-Teller (b) electronic configurations of $\mathrm{C}_{60}^{3-}$. $\left|\Phi_{i}\right\rangle$ are adiabatic orbitals. latter case is the consequence of three-dimensional trough on the lowest energy surface of $\mathrm{C}_{60}^{3-}$ [13]. Using the above estimations we conclude that the Jahn-Teller configuration is the lowest and the ground state spin is $S=1 / 2$, which is indeed found in Mott-Hubbard fullerides [6,7,5,13,14].

The three-dimensional trough on the lowest energy surface of $\mathrm{C}_{60}^{3-}$ leads to a complex nuclear dynamics, involving free rotations of Jahn-Teller deformations along three angular coordinates and harmonic vibrations along another degrees of freedom. The solutions for low-lying states in the strong coupling approximation are [13,14]:

$$
\begin{gathered}
\Psi(r, q, \alpha, \gamma, \vartheta, \varphi)=\Psi^{\mathrm{ad}}(r, \gamma, \vartheta, \varphi) \Psi_{n_{1}, n_{2}}^{\mathrm{vib}}(q, \alpha) \Psi_{L, M, K}^{\mathrm{rot}}(\gamma, \vartheta, \varphi), \\
E_{n_{1} n_{2} L K}=-E_{\mathrm{JT}}+\hbar \bar{\omega}_{H}\left(n_{1}+n_{2}+1\right) \\
+\frac{\hbar^{2}}{2 q_{0}^{2}}\left[\frac{1}{4}+L(L+1)-\frac{3}{4} K^{2}\right],
\end{gathered}
$$

where $r$ denotes electronic coordinates, $q$ is the amplitude of the $H_{g}$ vibrations and $\gamma, \vartheta, \varphi$ are three Euler angles specifying the rotation in the trough. $\Psi^{\text {ad }}$ is the adiabatic electronic function, describing the electronic state at given Euler coordinates (a Slater determinant of adiabatic orbitals, Fig. 1b); $\Psi^{\mathrm{vib}}$ describes two-dimensional vibrations across the trough; $\Psi_{L, M . K}^{\mathrm{rot}}$ is the rotational part, coinciding with the solution for a symmetric top with $K$ even. The ground state corresponds to $L=1, K=0$,

$$
\Psi_{1, M, 0}^{\mathrm{rot}}=\sqrt{\frac{2}{\pi}} Y_{1 M}(\vartheta, \varphi),
$$

and is therefore threefold degenerate. The nature of the ground state does not change when going beyond strong coupling approximation and when multiplet effects are taken into account $[13,14]$.

The lowest vibronic states in Eq. (1) correspond to excitations in the rotational sector at $n_{1}=n_{2}=0$. The rotational quantum can be estimated as $\hbar^{2} / 2 q_{0}^{2}=\frac{1}{4}\left(\hbar \bar{\omega}_{H}\right)^{2} / E_{\mathrm{JT}} \approx$ $\frac{1}{8} \hbar \bar{\omega}_{H} \approx 75 \mathrm{~cm}^{-1}$, where $q_{0}$ is the radius of the trough. The solutions (1) and (2) correspond to full delocalization of Jahn-Teller deformations in the trough, i.e., to the absence of static $H_{g}$ deformations. In a crystal they can only be localized (and the adiabatic orbitals fixed at some $\left.\gamma_{0}, \vartheta_{0}, \varphi_{0}\right)$ when the crystalline interactions mixing different rotational levels exceed the spacing between them [19]. In $\mathrm{Li}_{3}\left(\mathrm{NH}_{3}\right)_{6} \mathrm{C}_{60}$ the local crystal field at fullerene sites is of cubic symmetry and therefore the only source of localization of Jahn-Teller deformations could be the superexchange interaction between neighboring fullerenes and the vibrational intersite interaction (phonon dispersion). To estimate the superexchange amplitude [20], we take $t \approx W /(2 \cdot z)$, where $W=0.3 \mathrm{eV}$ is the LUMO bandwidth [5] and $z=8$ for a b.c.c lattice, and $U=1 \mathrm{eV}$ and obtain $4 t^{2} / U \approx 11 \mathrm{~cm}^{-1}$, which is much smaller than the estimated rotational quantum. On the other hand the frequency of interfullerene optical vibrations was found $\approx 40 \mathrm{~cm}^{-1}$ [1], and should be even smaller in ammoniated crystals, which again does not exceed the rotational quantum. We may conclude therefore that in $\mathrm{Li}_{3}\left(\mathrm{NH}_{3}\right)_{6} \mathrm{C}_{60}$ the fullerene 
anions will be in threefold degenerate vibronic ground states (2).

\section{Superexchange interaction between two $C_{60}^{3-}$ cubic sites}

In the Mott-Hubbard insulating regime the LUMO electrons are mainly localized at the fullerene sites and gain some kinetic energy via virtual hops to neighbour sites. The resulting partial electron delocalization depends on the relative orientation of the spins on the corresponding sites and is described by an effective superexchange Hamiltonian [20]. A systematic procedure for constructing superexchange Hamiltonians, based on the partioning of the total Hamiltonian into two terms, one acting within a chosen subspace of low-lying states and the other coupling different subspaces, was proposed long time ago [21] and applied for the derivation of the $t-J$ model. A more conventional approach [20] consists in partioning the total Hamiltonian into a term containing the sum of single-site contributions and another one corresponding to electron transfer between the LUMO orbitals of the neighbour fullerene sites, $\mathcal{H}_{\mathrm{t}}$. The single-site Hamiltonian is further divided into a term $\mathcal{H}_{0}$ containing the interactions depending on the total occupation number of sites, with an effective intrasite electron repulsion parameter $U$, and $\mathcal{H} \prime$ including the remaining intrafullerene interactions. Performing a unitary transformation which eliminates $\mathcal{H}_{\mathrm{t}}$ we obtain the following general expression for the effective Hamiltonian up to the second order after $1 / U[22,23]$ :

$\mathcal{H}_{\text {eff }}=\mathcal{H}_{0}+\mathcal{H}^{\prime}+\frac{1}{U} \mathcal{H}_{\mathrm{t}} \cdot \mathcal{H}_{\mathrm{t}}-\frac{1}{2 U^{2}}\left[\left[\mathcal{H}^{\prime}, \mathcal{H}_{\mathrm{t}}\right], \mathcal{H}_{\mathrm{t}}\right]$

This Hamiltonian acts in the space of the ground manifold of states with fixed number of electrons on sites. Since it is derived in the second order after intersite electron transfer, the superexchange part of this Hamiltonian (the last two terms) becomes a sum over pairs of sites which can be connected by $\mathcal{H}_{\mathrm{t}}$. In the absence of spin-orbit coupling on sites the contribution of each such pair $(i j)$ can be written in the form:

$\mathcal{H}_{i j}=K_{i j}+\left(\mathbf{S}_{i} \mathbf{S}_{j}+\frac{1}{4}\right) J_{i j}$,

describing the interaction between the states of the lowest terms ${ }^{\left(2 S_{i}+1\right)} \Gamma_{i}$ and ${ }^{\left(2 S_{j}+1\right)} \Gamma_{j}$ of the sites $i$ and $j$ respectively. The operators $K_{i j}$ and $J_{i j}$ act in the space of orbital indices of these terms and therefore represent matrices of dimension $\left|\Gamma_{i}\right| \cdot\left|\Gamma_{j}\right|$. In the case of orbitally nondegenerate sites, $K_{i j}$ and $J_{i j}$ become numbers and Eq. (4) reduces to the Heisenberg superexchange Hamiltonian [20]. In the case of twofold orbital degeneracy of sites, $K_{i j}$ and $J_{i j}$ are $4 \times 4$ matrices and can be rewritten via bilinear combinations of $\tilde{S}=1 / 2$ orbital pseudospins of sites, in which form Eq. (4) is known as KugelKhomskii Hamiltonian [9].

The electron transfer between the LUMO orbitals of neighbour fullerene sites in a b.c.c. lattice (Fig. 2) is described by $\mathcal{H}_{\mathrm{t}}$ where all diagonal $\left(t_{\gamma \gamma}\right)$ and all off-diagonal $\left(t_{\gamma \gamma^{\prime}}\right)$ transfer parameters are equal $(\gamma=x, y, z)$. The third term in (3) is the Anderson's antiferromagnetic contribution and the last term describes the effect of multiplet and Jahn-Teller splitting of $t_{1 u}$ shells on superexchange. The parameter $U$ entering these terms corresponds to an average $\mathrm{C}_{60}^{3-} \mathrm{C}_{60}^{3-} \rightarrow \mathrm{C}_{60}^{2-} \mathrm{C}_{60}^{4-}$ electron promotion energy and is estimated as $U=\frac{4}{3} U_{\perp}-\frac{1}{3} U_{\|}-\frac{2}{3} J+\frac{4}{3} E_{\mathrm{JT}}+V_{\mathrm{e}-\mathrm{h}}$. To obtain the superexchange Hamiltonian one should average $\mathcal{H}_{\text {eff }}$ over the vibronic ground states ${ }^{2} T_{1 u} \gamma$ of fullerene sites, Eqs. (1) and (2). This is done in two steps. First we average over the electronic coordinates entering the adiabatic functions $\Psi^{\text {ad }}$ and obtain for each pair of nearest neighbour sites an operator of Heisenberg type, Eq. (4) $\left(S_{i}=S_{j}=1 /\right.$ 2),

$$
\begin{aligned}
\mathcal{H}_{\mathrm{ex}}= & -2 \frac{t_{12}^{2}+t_{21}^{2}}{U}\left(1-\frac{2 E_{\mathrm{JT}}+J}{3 U}\right) \\
& -\frac{t_{13}^{2}+t_{31}^{2}}{U}\left(1+\frac{E_{\mathrm{JT}}-J}{3 U}\right) \\
& -\frac{t_{23}^{2}+t_{32}^{2}}{U}\left(1+\frac{E_{\mathrm{JT}}-7 J}{3 U}\right) \\
& -2 \frac{t_{33}^{2}}{U}\left(1+\frac{4 E_{\mathrm{JT}}-10 J}{3 U}\right)+\left[4 \frac{t_{33}^{2}}{U}\left(1+\frac{4 E_{\mathrm{JT}}-10 J}{3 U}\right)\right. \\
& \left.-2 \frac{t_{13}^{2}+t_{31}^{2}+t_{23}^{2}+t_{32}^{2}}{U} \frac{J}{U}\right]\left(\mathbf{S}_{i} \mathbf{S}_{j}+\frac{1}{4}\right),
\end{aligned}
$$

where the parameters $K_{i j}$ and $J_{i j}$ depend on six Euler angles of the two fullerene sites via the transfer parameters $t_{\gamma \gamma^{\prime}}$, where now $\gamma, \gamma^{\prime}=1,2,3$ denote the adiabatic orbitals on the site $i$ and $j$, respectively, in the order shown in Fig. 1. This Hamiltonian itself describes the exchange interaction in the case of static vibronic distortions on sites, when the six Euler angles become parameters. This is probably the case in noncubic ammoniated fullerides [6,7], and TDAE- $\mathrm{C}_{60}$ where fullerene sites are located in low-symmetry environments. In Eq. (5) the antiferromagnetic contribution is determined by the transfer parameter between half filled orbitals, $t_{33}$. Therefore the exchange is ferromagnetic for $t_{33}=0$, which is always possible to achieve by varying the Euler angles on sites. This seems to be the case in $\mathrm{K}_{3}\left(\mathrm{NH}_{3}\right) \mathrm{C}_{60}$. Indeed, the low temperature measurements show that the spins arrange in this compound antiferro-

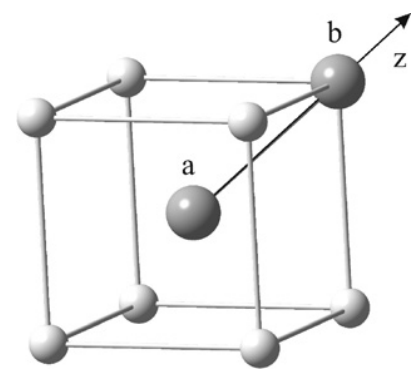

Fig. 2. Two nearest neighbour fullerene sites in a b.c.c. lattice. $z$ denotes the axis along which the total vibronic momentum of the pair is conserved. 
magnetically along the translational directions (similar orbitals on neighbour sites overlap) and ferromagnetically in perpendicular directions [7]. A similar mechanism was proposed to explain the ferromagnetism in TDAE- $\mathrm{C}_{60}$ [24].

In the present case the Euler angles are dynamical variables and we should further average the Hamiltonian (5) over wave functions (2). This leads to a superexchange Hamiltonian (4) where $K_{i j}$ and $J_{i j}$ are $9 \times 9$ matrices in the space of vibronic states of the two sites. The obtained Hamiltonian describes the spin-vibronic exchange interaction, which formally resembles the spin-orbital superexchange $[9,10]$ but acts in the space of vibronic rather than orbital states.

Given the threefold symmetry of nearest neighbour pairs, the intersite electron transfer is only nonzero between the $t_{1 u}$ orbitals with the same projection of the orbital momentum $(-1,0,1)$ on the axis of a given pair: $t_{0}=t_{x x}+2 t_{x y}, t_{1}=t_{-1}=t_{x x}-t_{x y}$. Similarly, the spin-vibronic superexchange will conserve the projection of the total vibronic momentum $I=M_{i}+M_{j}$ on the axis of the pair. It is convenient therefore to choose the quantization axis along the axis of the pair as shown in Fig. 2. The spin-vibronic spectrum for a single pair is shown in Fig. 3a. For comparison Fig. 3b shows the spin-orbital spectrum of a corresponding dimer containing threefold orbitally degenerate sites, the $t_{2 g}^{1}-t_{2 g}^{1}$ model (similar to the $\mathrm{p}^{1}-\mathrm{p}^{1}$ model [23]). The corresponding Hamiltonian was obtained by averaging the $\mathcal{H}_{\text {eff }}$ in Eq. (3), containing the same parameters (except for the Jahn-Teller energy which is absent in the spin-orbital model), over threefold degenerate orbitals of sites. The obtained energy levels are characterized by the conserved projection of the total orbital momentum on the axis of the pair, which we denote also by $I$. One can see two main differences in these spectra. First, the splitting of the spin levels in the spin-vibronic case is 3-4 times smaller than in the spin-orbital case. Second, while the ground state of the spin-orbital pair can be both ferro and antiferromagnetic, the ground state of the spin-vibronic pair is always antiferromagnetic. This is supported by the antiferromagnetic susceptibility observed in $\mathrm{Li}_{3}\left(\mathrm{NH}_{3}\right)_{6} \mathrm{C}_{60}[5]$.

\section{Superexchange in extended lattice: 1 . Ordering in the spin sector}

In order to describe the effect of superexchange interaction in an extended lattice we use a boson representation of $K_{i j}$ and $J_{i j}$ in (4) following the approach adopted for spinorbital models [25]. We consider again two neighbour fullerene sites and choose the axis of the pair as quantization axis for orbital and vibronic moments (Fig. 2). Keeping for simplicity the main part of superexchange interaction - the third term in Eq. (3) (the terms arising from $\mathcal{H}^{\prime}$ do not change qualitatively the results as Fig. 3a suggests and as shown elsewhere [22]), we obtain (in units of $4 t_{0}^{2} / 50 U$ ):

$$
\begin{aligned}
J_{i j}= & \left(8+9 \eta^{2}\right)+\left(3 \eta^{2}-4\right)\left(n_{0}^{a}+n_{0}^{b}\right)+\left(2+\eta^{2}\right) n_{0}^{a} n_{0}^{b} \\
& +\frac{1}{\pi}\left[\eta^{2} a_{1}^{\dagger} a_{-1} b_{-1}^{\dagger} b_{1}\right. \\
& \left.+\frac{\eta}{2}\left(a_{1}^{\dagger} a_{0}-a_{0}^{\dagger} a_{-1}\right)\left(b_{0}^{\dagger} b_{1}-b_{-1}^{\dagger} b_{0}\right)+\text { h.c. }\right], \\
K_{i j}= & \left(-9+2 \eta^{2}\right) n_{0}^{a} n_{0}^{b}+\frac{1}{\pi}\left[2 \eta^{2} a_{1}^{\dagger} a_{-1} b_{-1}^{\dagger} b_{1}\right. \\
& \left.+\eta\left(a_{1}^{\dagger} a_{0}-a_{0}^{\dagger} a_{-1}\right)\left(b_{0}^{\dagger} b_{1}-b_{-1}^{\dagger} b_{0}\right)+\text { h.c. }\right],
\end{aligned}
$$

where $a_{M}$ and $b_{M}$ are bosonic operators (analogues of orbitons in spin-orbital models [25]) corresponding to vibronic states (2) of the first and second fullerene site, respectively, constrained by the conditions $n_{0}^{a}+n_{1}^{a}+n_{-1}^{a}=1$ and

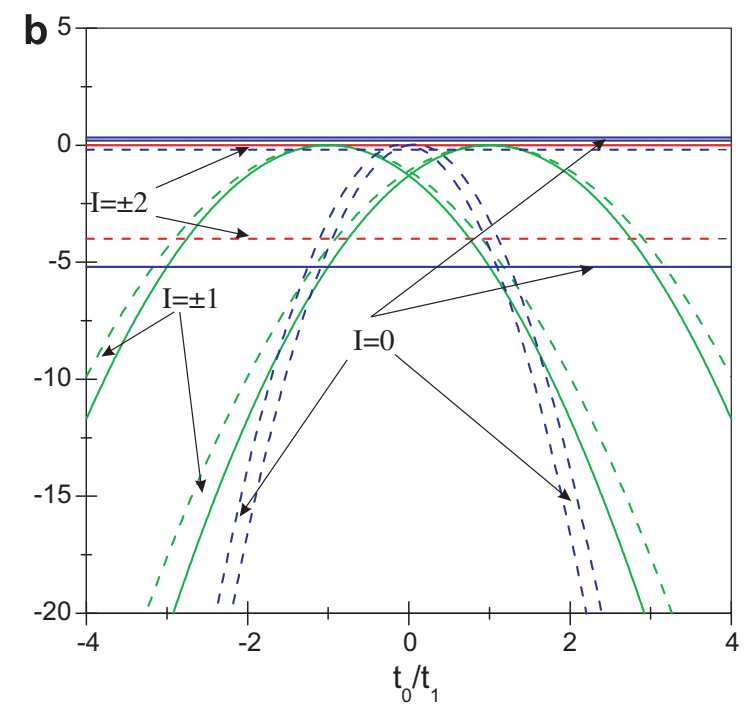

Fig. 3. Spin-vibronic spectrum for $\mathrm{C}_{60}^{3-}-\mathrm{C}_{60}^{3-}$ at $J / U=0.05, E_{\mathrm{JT}}^{3} / U=0.15$ (a) and spin-orbital spectrum for a $t_{2 g}^{1}-t_{2 g}^{1}$ pair at $J / U=0.1$ (b) in a b.c.c. lattice. Solid lines correspond to $S=1$ and dashed lines to $S=0$ ( $S$ is the spin of the pair). (For interpretation of the references to color in this figure legend, the reader is referred to the web version of this paper.) 
$n_{0}^{b}+n_{1}^{b}+n_{-1}^{b}=1 ; \eta=t_{1} / t_{0}$ and the constant term in the expression for $K_{i j}$ was omitted.

The constant term in $J_{i j}$ is dominant for any $\eta$, which means that the coupling between nearest neighbour spins is always antiferromagnetic, in full agreement with magnetic susceptibility data for $\mathrm{Li}_{3}\left(\mathrm{NH}_{3}\right)_{6} \mathrm{C}_{60}$ [5]. In the ordered phase, the spin moments of the two antiferromagnetic sublattices will be almost unreduced with respect to the corresponding Heisenberg model, given the relative smallness of vibronic terms in $J_{i j}$. This behaviour differs much from the case of crystals with orbitally degenerate sites $\left(t_{2 g}^{1}-t_{2 g}^{1}\right.$ pairs) both in perovskites (titanates) $[25,26]$ and b.c.c. lattices [22]. In the last case the interaction between spins is strongly dependent on orbital populations and can be equally ferro and antiferromagnetic (this is seen also in Fig. 3b), with spin moments strongly reduced by orbital fluctuations in ordered phases.

The specific feature of spin-vibronic superexchange comes from the delocalization of the adiabatic orbitals over all three $t_{1 u} \gamma$ orbitals in each vibronic state (2). As a result the electron transfer between singly occupied adiabatic orbitals of the neighbour sites $\left(\left|\Phi_{3}\right\rangle\right.$ in Fig. 1) is never zero. Other feature of $(6)$ is the reduction of the superexchange amplitude with respect to $4 t^{2} / U$. Indeed for various values of transfer parameters we obtain for the superexchange amplitude:

$$
\begin{aligned}
& t_{0}=0: \frac{9}{50} \frac{4 t_{1}^{2}}{U} \\
& t_{1}=0: \frac{8}{50} \frac{4 t_{0}^{2}}{U} \\
& t_{1}=t_{0}: \frac{17}{50} \frac{4 t_{0}^{2}}{U}
\end{aligned}
$$

This is again the consequence of delocalization of the adiabatic orbitals $\left|\Phi_{3}\right\rangle$ in the dynamical vibronic states (2) and corresponds to vibronic reduction of superexchange interactions. It is just an example of Ham's reduction of electronic operators in Jahn-Teller systems [12]. Such a reduction of exchange interaction is indeed observed in $\mathrm{Li}_{3}\left(\mathrm{NH}_{3}\right)_{6} \mathrm{C}_{60}$ : from the experimental Weiss constant $\theta=-15.2 \mathrm{~K}$ [5] one derives the mean-field estimate for the exchange parameter of $5.3 \mathrm{~cm}^{-1}$ which is twice smaller than the previous estimate based on the LUMO bandwidth.

\section{Superexchange in extended lattice: 2 . Ordering in the vibronic sector}

Consider now the vibronic sector of superexchange interaction. Since $J_{i j}>K_{i j}$ the spin order sets in first and therefore the ordering in vibronic sector takes place on the background of antiferromagnetic spin order. As Eq. (6) shows the term $K_{i j}$ is an operator even in the absence of Hund's rule coupling on sites. This means that the vibronic order is stabilized already for classical (Neel) order of spins. In contrast $K_{i j}$ reduces to a constant in spin-orbital models with $t_{2 g}^{1}$ sites, when $J=0$, for both simple cubic
[26] and b.c.c. lattices [22], which means that their classical Neel state, $\left\langle\mathbf{S}_{i} \mathbf{S}_{j}\right\rangle=-1 / 4$, is infinitely degenerate in the orbital sector. As a consequence the orbital order is stabilized in these models only by spin fluctuations.

Averaging (4) over the antiferromagnetic spin ordered state we obtain the vibronic Hamiltonian:

$\mathcal{H}_{\text {vibr }}=\sum_{\langle i j\rangle}\left(K_{i j}+\xi J_{i j}\right)$,

where the bosonic operators in each term are defined with respect to the quantization axis of the corresponding pair and $\xi=\left\langle\mathbf{S}_{i} \mathbf{S}_{j}\right\rangle_{\text {af }}+1 / 4$. It results that the terms $n_{0}^{a} n_{0}^{b}$ in (6) give the main contribution to (7) when $\left(t_{1} / t_{0}\right)^{2}$ is not too close to $(9-2 \xi) /(2+\xi)$. Considering this condition fulfilled we further keep only these terms. Next we introduce the vibronic momentum operators $I_{x}=i\left(a_{z}^{\dagger} a_{y}-a_{y}^{\dagger} a_{z}\right)$, $I_{y}=i\left(a_{x}^{\dagger} a_{z}-a_{z}^{\dagger} a_{x}\right), I_{z}=i\left(a_{y}^{\dagger} a_{x}-a_{x}^{\dagger} a_{y}\right)$ and the quadrupole operators $T_{x}=-\left(I_{y} I_{z}+I_{z} I_{y}\right), \quad T_{y}=-\left(I_{x} I_{z}+I_{z} I_{x}\right), \quad T_{z}=$ $-\left(I_{x} I_{y}+I_{y} I_{x}\right)$ for each fullerene site (they are similar to the case of $t_{2 g}^{1}$ orbital degeneracy [25]). Then the vibronic Hamiltonian can be rewritten in the form:

$\mathcal{H}_{\text {vibr }}=\frac{4}{9} C \sum_{\mathbf{i}}\left[1+\sum_{j=0}^{3}\left(\mathbf{T}^{\mathbf{i}} \mathbf{e}_{j}\right)\left(\mathbf{T}^{\mathbf{i}+\mathbf{e}_{j}} \mathbf{e}_{j}\right)\right]$,

where $C=\left(4 t_{0}^{2} / 50 U\right)\left[(9-2 \xi)-(2+\xi) \eta^{2}\right], \mathbf{e}_{1-3}$ are b.c.c. unit vectors (in units of cubic lattice constant) and $\mathbf{e}_{0}$ is minus their sum (these vectors form a tetrahedral star around each site $\mathbf{i})$.

In the case $C<0$ the quadrupole moments order ferromagnetically (order I) along one of the trigonal axes of the crystal (Fig. 4a) with the interaction energy $E_{\text {vibr }}=(4 / 9) C$ per site. For positive $C$ the lowest configuration corresponds to antiferro ordering (II) of quadrupole moments along one of tetragonal axes (Fig. 4b) with $E_{\text {vibr }}=0$. We stress that these orders are stabilized already at the classical level. The phases shown in Fig. 4 have the vibronic states on sites: $a=\left(a_{x}+a_{y}+a_{z}\right) / \sqrt{3}$ for ferro and $a_{1,2}=\left(a_{x} \mp a_{y}\right) / \sqrt{2}$ for the two sublattices of antiferro order, where $a_{x}, a_{y}, a_{z}$ correspond to real combinantions of the vibronic states (2). It results therefore that in both these orders the vibronic moments on sites are completely quenched (the same for orbital moments, of course).
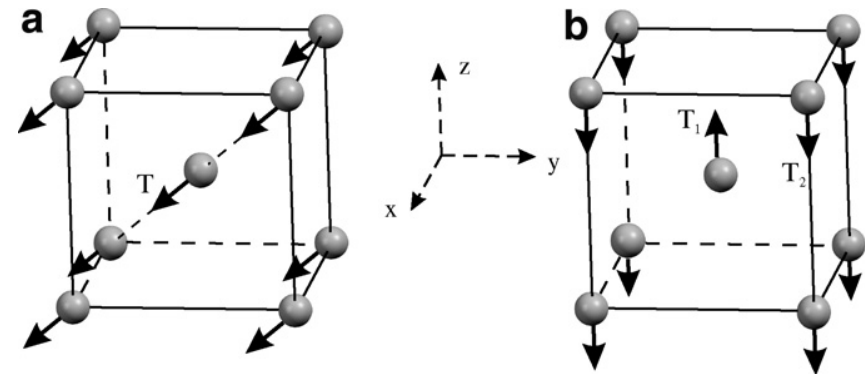

Fig. 4. Vibronic quadrupole ordered states: (a) ferro type (I), $T=(-2 / 3$, $-2 / 3,-2 / 3)$; (b) antiffero type (II), $T_{1}=(0,0,1), T_{2}=(0,0,-1)$. 


\section{Collective vibronic excitations in the ordered phases}

To investigate the vibronic excitation spectrum, we introduce on each site, following Ref. [25], two states orthogonal to the condensed state $a: \alpha=\left(a_{x}-a_{y}\right) / \sqrt{2}$, $\beta=\left(2 a_{z}-a_{x}-a_{y}\right) \sqrt{6}$ for order I and $\alpha_{1,2}=\left(a_{x} \pm a_{y}\right) / \sqrt{2}$, $\beta_{1,2}=a_{z}$ for two sublattices of order II.

The linearized Hamiltonian for the ferro phase has the form (in units of $8|C| / 9$ ):

$$
\begin{aligned}
\mathcal{H}_{\mathrm{VW}}^{\mathrm{I}}= & \sum_{\mathbf{i}}\left\{2\left(n_{\alpha}^{\mathrm{i}}+n_{\beta}^{\mathrm{i}}\right)-\frac{1}{9}\left[T_{\alpha}^{\mathrm{i}} T_{\alpha}^{\mathrm{i}+\mathbf{e}_{3}}+\left(\frac{1}{2} T_{\alpha}^{\mathrm{i}}-\frac{\sqrt{3}}{2} T_{\beta}^{\mathrm{i}}\right)\right.\right. \\
& \times\left(\frac{1}{2} T_{\alpha}^{\mathrm{i}+\mathbf{e}_{1}}-\frac{\sqrt{3}}{2} T_{\beta}^{\mathrm{i}+\mathbf{e}_{1}}\right)+\left(\frac{1}{2} T_{\alpha}^{\mathrm{i}}+\frac{\sqrt{3}}{2} T_{\beta}^{\mathrm{i}}\right) \\
& \left.\left.\times\left(\frac{1}{2} T_{\alpha}^{\mathrm{i}+\mathbf{e}_{2}}+\frac{\sqrt{3}}{2} T_{\beta}^{\mathrm{i}+\mathbf{e}_{2}}\right)\right]\right\}
\end{aligned}
$$

where $T_{\alpha} \approx-\left(\beta+\beta^{\dagger}\right)$ and $T_{\beta} \approx-\left(\alpha+\alpha^{\dagger}\right)$. The form of this Hamiltonian is isomorphic with the orbital superexchange terms found in the spin ferromagnetic phase in the cuprate model, where $T_{\alpha}$ and $T_{\beta}$ corresponded to orbital pseudospin operators of twofold orbitally degenerate $\mathrm{Cu}(\mathrm{II})$ sites [27]. Despite different physical content, the equivalent form of these Hamiltonians is dictated by symmetry. Indeed, they reflect the only way in which one can write an invariant, with respect to rotations around the trigonal axis, for scalar products of two-dimensional vectors $\left(T_{\alpha}, T_{\beta}\right)$.

Here the Hamiltonian (9) describes two branches of optical magnons of vibronic origin with excitation energies

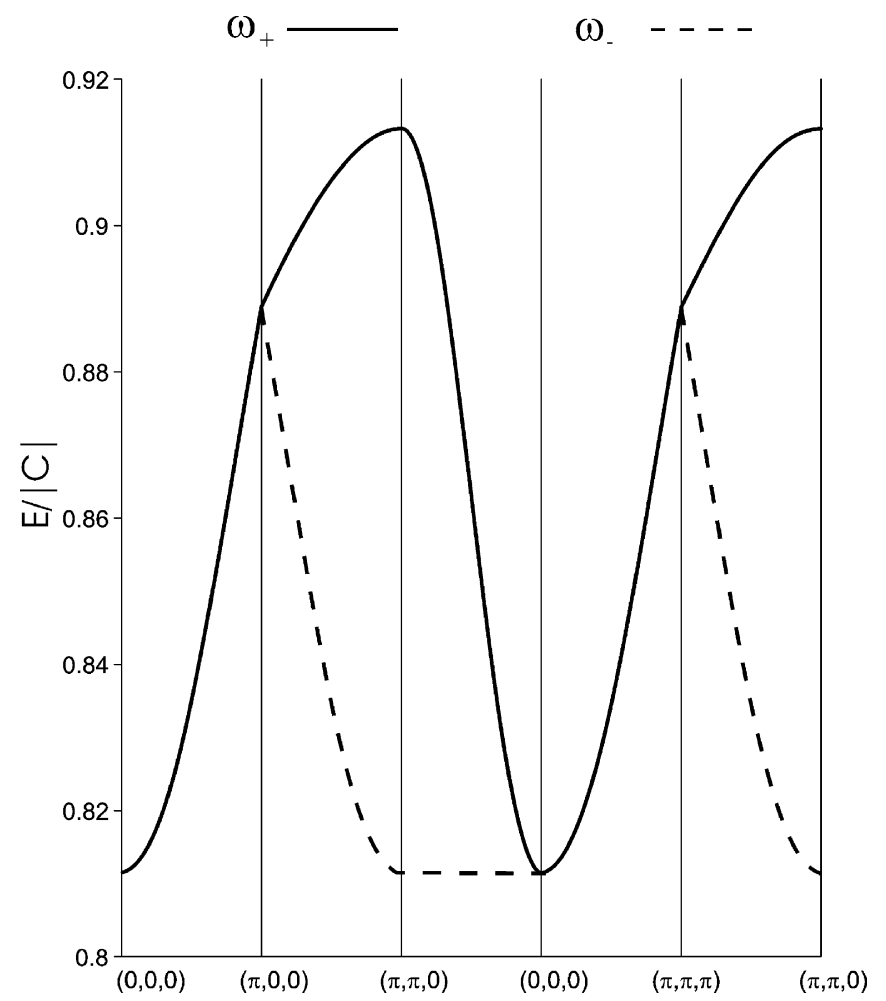

Fig. 5. Dispersion of vibronic excitations in the ferromagnetic phase (I). $\omega_{ \pm}^{\mathbf{k}}=\sqrt{1+\left(-C_{a} \pm \sqrt{C_{\theta}^{2}+C_{\epsilon}^{2}}\right) / 6}$, where $C_{a}=\left(\cos k_{1}+\right.$ $\left.\cos k_{2}+\cos k_{3}\right) / 3, \quad C_{\theta}=\left(2 \cos k_{3}-\cos k_{1}-\cos k_{2}\right) / 6, \quad C_{\epsilon}=$ $\sqrt{3}\left(\cos k_{1}-\cos k_{2}\right) / 6$ and $k_{i}$ are projections of the momentum along the corresponding $\mathbf{e}_{i}$. The obtained spectrum of vibronic excitations (Fig. 5) has a cubic symmetry and is characterized by a relatively large gap and small dispersion. This results in a negligible number of bosons not in the condensate:

$\left\langle n_{\alpha}+n_{\beta}\right\rangle=\frac{1}{4 N} \sum_{\mathbf{k}}\left[\frac{\left(\omega_{+}^{\mathbf{k}}-1\right)^{2}}{\omega_{+}^{\mathbf{k}}}+\frac{\left(\omega_{-}^{\mathbf{k}}-1\right)^{2}}{\omega_{-}^{\mathbf{k}}}\right] \approx 0.001$.

Accordingly the order parameter in this phase $\left\langle T_{z}\right\rangle=\left\langle T_{x}\right\rangle=\left\langle T_{y}\right\rangle=-\left(2 / 3-\left\langle n_{\alpha}+n_{\beta}\right\rangle\right)$ is almost unreduced with respect to the classical equilibrium value (Fig. 4a).

The linearized Hamiltonian for the antiferro phase is of the form (in units of $8 C / 9$ ):

$\mathcal{H}_{\mathrm{VW}}^{\mathrm{II}}=\sum_{i}\left(2 n_{\alpha}^{i}+n_{\beta}^{i}\right)$,

i.e., involving strictly local excitations. Thus the order parameters of both vibronic phases turn out to be almost unaffected by quantum fluctuations, which is the consequence of their classical origin. The parameter $C$ is much smaller than the constant part of $J_{i j}$ in (6), which means that the vibronic order sets in at a much lower temperature than the spin order.

\section{Discussion and conclusions}

In order to assess what vibronic order is realized in $\mathrm{Li}_{3}\left(\mathrm{NH}_{3}\right)_{6} \mathrm{C}_{60}$ we estimate the ratio between the two transfer parameters in orientationally ordered b.c.c. fullerene crystals taking into account only the direct overlap of $t_{1 u}$ orbitals of nearest neighbour fullerenes. The overlap between carbon orbitals was calculated within Harrison [28] and Wolfsberg-Helmholz approximations. Fig. 6 shows that for the lattice constant corresponding to $\mathrm{Li}_{3}\left(\mathrm{NH}_{3}\right)_{6} \mathrm{C}_{60}, \eta^{2} \approx 14(\mathrm{H})$ and $9(\mathrm{WH})$. These numbers are well above the critical value $(9-2 \xi) /(2+\xi) \approx 4.5$

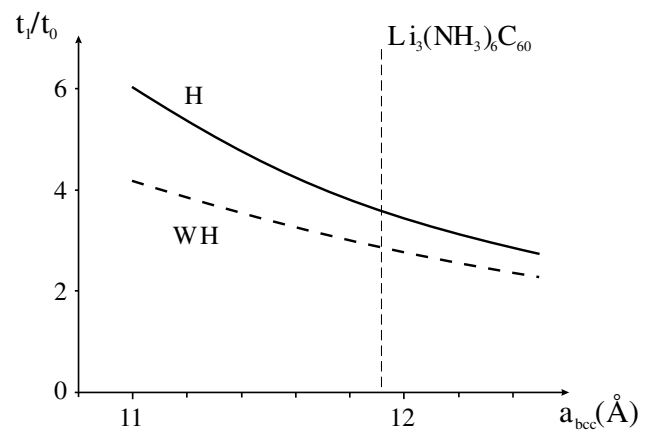

Fig. 6. Ratio of transfer parameters for LUMO orbitals of nearest neighbour fullerenes in a b.c.c. lattice, calculated within Harrison and Wolfsberg-Helmholz approximations. 
$(\xi \approx 0)$ thus justifying the approximation made when obtaining Eq. (8). From these estimations we infer that $C<0$ implying the vibronic order of type I in the ground state of this fulleride.

Concerning the approximations made in deriving the above model one should stress that the threefold vibronic degeneracy of sites is a robust feature of $\mathrm{C}_{60}^{3-}$ if the vibronic dynamics is unquenched according to our estimations. Actually the major approximation in our model is the neglect of the contribution to the superexchange interaction which comes through the exchange of virtual phonons [29]. It is known that this contribution is generally important in concentrated Jahn-Teller systems although it is not clear whether it is still so in the case dynamical JahnTeller effect on sites. We will address this question in the future work [22].

In conclusion, the ground state and low-lying excitations in $\mathrm{Li}_{3}\left(\mathrm{NH}_{3}\right)_{6} \mathrm{C}_{60}$ (and probably other Mott-Hubbard cubic fullerides) are governed by spin-vibronic superexchange between neighbour fullerene sites. This interaction formally resembles the spin-orbital superexchange but involves degenerate vibronic instead of orbital states on sites. The predictions of the spin-vibronic model are in line with available magnetic data for $\mathrm{Li}_{3}\left(\mathrm{NH}_{3}\right)_{6} \mathrm{C}_{60}$. We suggest that a quadrupole vibronic order develops at a temperature much lower than the temperature of spin ordering transition, which could be tested in future experiments.

\section{Acknowledgements}

I would like to thank Andrzej Oleś and Michael Kaplan for useful discussions.

\section{References}

[1] O. Gunnarsson, Rev. Mod. Phys. 69 (1997) 575.

[2] M.J. Rosseinsky, Chem. Mater. 10 (1998) 2665.

[3] M. Imada, A. Fujimori, Y. Tokura, Rev. Mod. Phys. 70 (1998) 1039.
[4] Y. Tokura, N. Nagaosa, Science 288 (2000) 462.

[5] P. Durand, G.R. Darling, Y. Dubitsky, A. Zaopo, M.J. Rosseinsky, Nat. Materials 2 (2003) 605.

[6] Y. Iwasa, H. Shimoda, T.T. Palstra, Y. Maniwa, O. Zhou, T. Mitani, Phys. Rev. B 53 (1996) R8836.

[7] S. Margadonna, K. Prassides, J. Solid State Chem. 168 (2002) 639.

[8] L.F. Chibotaru, Phys. Rev. Lett. 94 (2005) 186405.

[9] K.I. Kugel, D.I. Khomskii, Sov. Phys. Usp. 25 (1982) 231.

[10] A.M. Oleś, M. Cuoco, N.B. Perkins, in: F. Mancini (Ed.), Lectures of the Physics of Highly Correlated Electron Systems IV, AIP Conf. Proc., vol. 527, New York, 2000.

[11] M.C.M. O'Brien, Phys. Rev. 187 (1969) 407.

[12] I.B. Bersuker, V.Z. Polinger, Vibronic Interactions in Molecules and Crystals, Springer, Berlin, 1989.

[13] A. Auerbach, N. Manini, E. Tosatti, Phys. Rev. B 49 (1994) 12998; N. Manini, A. Auerbach, E. Tosatti, Phys. Rev. B 49 (1994) 13008.

[14] M.C.M. O’Brien, Phys. Rev. B 53 (1996) 3775; C.C. Chancey, M.C.M. O'Brien, The Jahn-Teller Effect in $\mathrm{C}_{60}$ and Other Icosahedral Complexes, Princeton University Press, 1997.

[15] O. Gunnarsson, H. Handschuh, P.S. Bechthold, B. Kessler, G. Ganteför, W. Eberhardt, Phys. Rev. Lett. 74 (1995) 1875.

[16] Using the vibronic constants from quantum chemistry calculations $[1,17]$ gives higher values of $\bar{\omega}_{H}$.

[17] N. Manini, A. Dal Corso, M. Fabrizio, E. Tossati, Philos. Mag. B 81 (2001) 793.

[18] J.E. Han, O. Gunnarsson, V.H. Crespi, Phys. Rev. Lett. 90 (2003) 167006.

[19] The first and second rotational excited states lie at one and six rotational quanta, respectively [14].

[20] P.W. Anderson, Phys. Rev. 115 (1959) 2.

[21] K.A. Chao, J. Spalek, A.M. Oleś, J. Phys. C 10 (1977) L271; Phys. Rev. B 18 (1978) 3453.

[22] L.F. Chibotaru, unpublished.

[23] A. Ceulemans, L.F. Chibotaru, G.A. Heylen, K. Pierloot, L.G. Vanquickenborne, Chem. Rev. 100 (2000) 787.

[24] T. Kawamoto, Solid State Commun. 101 (1997) 231.

[25] G. Khaliullin, S. Okamoto, Phys. Rev. Lett. 89 (2002) 167201; Phys. Rev. B 68 (2003) 205109.

[26] G. Khaliullin, S. Maekawa, Phys. Rev. Lett. 85 (2000) 3950

[27] A.M. Oleś, L.F. Feiner, J. Zaanen, Phys. Rev. B 61 (2000) 6257; J. van den Brink, P. Horsch, F. Mack, A.M. Oleś, Phys. Rev. B 59 (1999) 6795.

[28] O. Gunnarsson, S.C. Erwin, E. Koch, R.M. Martin, Phys. Rev. B 57 (1998) 2159.

[29] M.D. Kaplan, B.G. Vekhter, Cooperative Phenomena in Jahn-Teller Crystals, Plenum Press, New York, 1995. 